\title{
THE STABLE MONOMORPHISM CATEGORY OF A FROBENIUS CATEGORY
}

\author{
XiaO-Wu Chen
}

\begin{abstract}
For a Frobenius abelian category $\mathcal{A}$, we show that the category $\operatorname{Mon}(\mathcal{A})$ of monomorphisms in $\mathcal{A}$ is a Frobenius exact category; the associated stable category $\underline{\operatorname{Mon}}(\mathcal{A})$ modulo projective objects is called the stable monomorphism category of $\mathcal{A}$. We show that a tilting object in the stable category $\mathcal{A}$ of $\mathcal{A}$ modulo projective objects induces naturally a tilting object in $\underline{\operatorname{Mon}}(\mathcal{A})$. We show that if $\mathcal{A}$ is the category of (graded) modules over a (graded) self-injective algebra $A$, then the stable monomorphism category is triangle equivalent to the (graded) singularity category of the (graded) $2 \times 2$ upper triangular matrix algebra $T_{2}(A)$. As an application, we give two characterizations to the stable category of Ringel-Schmidmeier.
\end{abstract}

\section{Introduction}

Let $\mathcal{A}$ be an abelian category. Denote by $\operatorname{Mor}(\mathcal{A})$ the category of morphisms in $\mathcal{A}$ ([3, p.101]): the objects are morphisms in $\mathcal{A}$ and the morphisms are given by commutative squares in $\mathcal{A}$. It is an abelian category ([17, Proposition 1.1]). We are mainly concerned with the full subcategory $\operatorname{Mon}(\mathcal{A})$ of $\operatorname{Mor}(\mathcal{A})$ consisting of monomorphisms in $\mathcal{A}$, which is called the monomorphism category of $\mathcal{A}$. It is an additive subcategory of $\operatorname{Mor}(\mathcal{A})$ which is closed under extensions, thus it becomes an exact category in the sense of Quillen ([22, Appendix A]).

In the case that the abelian category $\mathcal{A}$ is the module category over a ring, the monomorphism category $\operatorname{Mon}(\mathcal{A})$ is known as the submodule category. Recently it is studied intensively by Ringel and Schmidmeier $([34,35,36])$. If the ring is $\mathbb{Z} /\left(q^{p}\right)$ with $p \geq 2$ and $q$ a prime number, the study of the submodule category goes back to Birkhoff ([8]; see also [1]). The case that the ring is $k[t] /\left(t^{p}\right)$ with $k$ a field is studied by Simson ([37]) and also by Beligiannis ([7]). In this case, the study of indecomposable objects in $\operatorname{Mon}(\mathcal{A})$ shows an example of the typical trichotomy phenomenon "finite/tame/wild" in the representation theory of finite dimensional algebras, where the trichotomy depends on the parameter $p$; see [36, Section 6]. Moreover, the case where the abelian category $\mathcal{A}$ is given by the graded module category over the graded algebra $k[t] /\left(t^{p}\right)$ with $\operatorname{deg} t=1$ plays an important role in [36]; in this case, the

Received by the editors December 11, 2009. Revision received May 21, 2010.

1991 Mathematics Subject Classification. 18E30, 13E10, $18 \mathrm{G} 25$.

Key words and phrases. monomorphism category, tilting object, derived category, Gorensteinprojective modules, singularity category.

The author is supported by Special Foundation of President of The Chinese Academy of Sciences (No.1731112304061) and National Natural Science Foundation of China (No.10971206). 
monomorphism category $\operatorname{Mon}(\mathcal{A})$ is denoted by $\mathcal{S}(\widetilde{p})$. It is a Frobenius exact category ([27]; also see Lemma 2.1 and compare [22, Section 5]). Then by [18, Chapter I, Theorem 2.6] its stable category $\underline{\mathcal{S}}(\widetilde{p})$ modulo projective objects is triangulated. A very recent and remarkable result due to Kussin, Lenzing and Meltzer claims that the stable category $\underline{\mathcal{S}}(\widetilde{p})$ is triangle equivalent to the stable category of vector bundles on the weighted projective lines of type $(2,3, p)$; see [27]. Recall that a similar trichotomy phenomenon "domestic/tubular/wild" occurs in the classification of indecomposable vector bundles on the weighted projective lines of type $(2,3, p)$, while the trichotomy again depends on the parameter $p$; see $[29,26]$. In this paper, we will call the triangulated category $\underline{\mathcal{S}}(\widetilde{p})$ the stable category of Ringel-Schmidmeier.

The present paper studies the monomorphism category $\operatorname{Mon}(\mathcal{A})$ of a Frobenius abelian category $\mathcal{A}$, in particular, the stable category $\mathcal{A}$ modulo projective objects is triangulated. We show that $\operatorname{Mon}(\mathcal{A})$ is a Frobenius exact category and then the stable category $\operatorname{Mon}(\mathcal{A})$ modulo projective objects is triangulated; it is called the stable monomorphism category of $\mathcal{A}$. Recently this category is also studied by Iyama, Kato and Miyachi ([21]). Observe that the triangulated categories above are algebraical in the sense of Keller. We have a well-behaved notion of tilting object for an algebraical triangulated category $([24])$. We prove that a tilting object in $\mathcal{A}$ induces naturally a tilting object in $\underline{\operatorname{Mon}}(\mathcal{A})$; see Theorem 3.2. Moreover, if the category $\mathcal{A}$ is the (graded) module category over a (graded) self-injective algebra $A$, we relate the category $\underline{\operatorname{Mon}}(\mathcal{A})$ to the category of (graded) Gorenstein-projective modules and then to the (graded) singularity category of the $2 \times 2$ upper triangular matrix algebra $T_{2}(A)$ of $A$ (for $T_{2}(A)$, see [17, p.115] and [3, Chapter III, Section 2]); see Theorem 4.1. We are inspired by a computational result by Li and Zhang on Gorenstein-projective modules ([30]; compare [7, 21]). Here, the Gorenstein-projective module is in the sense of Enochs and Jenda ([16, Chapter 10]), and the singularity category is in the sense of Orlov ([32, 33]; compare [10, 19]).

Combining all these together, we give two characterizations to the stable category $\underline{\mathcal{S}}(\widetilde{p})$ of Ringel-Schmidmeier. We characterize the stable category $\mathcal{S}(\widetilde{p})$ as the bounded derived category of $T_{2}\left(k \mathbb{A}_{p-1}\right) \simeq k \mathbb{A}_{2} \otimes_{k} k \mathbb{A}_{p-1}$; see Corollary 3.4. Here, for each $n \geq$ $1, \mathbb{A}_{n}$ is the linear quiver with $n$ vertices and linear orientation, and $k \mathbb{A}_{n}$ is the path algebra. We characterize the stable category $\mathcal{S}(\widetilde{p})$ as the graded singularity category of $T_{2}(k)[t] /\left(t^{p}\right)$, where the algebra $T_{2}(k)[t] /\left(t^{p}\right)$ is graded such that $\operatorname{deg} T_{2}(k)=0$ and $\operatorname{deg} t=1$; see Corollary 4.7.

For the convention, throughout we fix a commutative artinian ring $R$. All artin algebras are artin $R$-algebras, and all categories and functors are $R$-linear. For an artin algebra $A$, denote by $\bmod A$ the category of finitely generated right $A$-modules and by $\operatorname{proj} A$ the full subcategory consisting of projective modules. We denote by $A_{A}$ and ${ }_{A} A$ the right and left regular modules of the artin algebra $A$, respectively. For triangulated categories and derived categories, we refer to [20, 18, 23, 24].

\section{Monomorphism Category}

Let $\mathcal{A}$ be a Frobenius abelian category. Thus $\mathcal{A}$ has enough projective objects and enough injective objects, and the class of projective objects coincides with the class of injective objects. Denote by $\mathcal{P}$ the full subcategory of $\mathcal{A}$ consisting of projective 
objects. Denote by $\mathcal{A}$ the stable category of $\mathcal{A}$ modulo $\mathcal{P}$ : the objects are the same as $\mathcal{A}$, and the morphism spaces are factors of the morphism spaces in $\mathcal{A}$ modulo those factoring through projective objects ([3, p.101]). The stable category $\mathcal{A}$ is a triangulated category such that its shift functor is given by the quasi-inverse of the syzygy functor on $\mathcal{A}$ and triangles are induced by short exact sequences in $\mathcal{A}$; for details, see [18, Chapter I, Section 2].

Recall that $\operatorname{Mor}(\mathcal{A})$ is the category of morphisms in $\mathcal{A}$ : the objects are morphisms $\alpha: A \rightarrow B$ in $\mathcal{A}$ and the morphisms are commutative squares in $\mathcal{A}$, that is, of the form $(f, g): \alpha \rightarrow \alpha^{\prime}$ where $f: A \rightarrow A^{\prime}$ and $g: B \rightarrow B^{\prime}$ are morphisms in $\mathcal{A}$ such that $\alpha^{\prime} \circ f=g \circ \alpha$ (compare [3, p.101]). For an object $\alpha: A \rightarrow B$ in $\operatorname{Mor}(\mathcal{A})$, we write $s(\alpha)=A$ and $t(\alpha)=B$, which are called the source and target of $\alpha$, respectively. Note that $\operatorname{Mor}(\mathcal{A})$ is an abelian category such that a sequence $\alpha^{\prime} \rightarrow \alpha \rightarrow \alpha^{\prime \prime}$ is exact if and only if the induced sequences of sources and targets are exact in $\mathcal{A}$ ([17, Corollary $1.2])$.

Recall that an exact category in the sense of Quillen is an additive category together with an exact structure, that is, a distinguished class of ker-coker sequences, which are called conflations, subject to certain axioms. Recall that a full additive subcategory of an abelian category which is closed under extensions has a natural exact structure such that conflations are just short exact sequences with terms in the subcategory ([22, Appendix A] and [23, Section 4]). Moreover, there is a notion of Frobenius exact category and the associated stable category modulo projective objects is still triangulated; compare [18, p.10-11], [22, subsection 1.2 b)] and [23, Section 6].

Recall that our main concern is the monomorphism category $\operatorname{Mon}(\mathcal{A})$, which is the full subcategory of $\operatorname{Mor}(\mathcal{A})$ consisting of monomorphisms in $\mathcal{A}$. We will consider the following two functors: the first functor $i_{1}: \mathcal{A} \rightarrow \operatorname{Mon}(\mathcal{A})$ is defined such that $i_{1}(A)=0 \rightarrow A$ and $i_{1}(f)=(0, f)$ where $A$ is an object and $f$ is a morphism in $\mathcal{A}$; the second $i_{2}: \mathcal{A} \rightarrow \operatorname{Mon}(\mathcal{A})$ is defined such that $i_{2}(A)=A \stackrel{\mathrm{Id}_{A}}{\rightarrow} A$ and $i_{2}(f)=(f, f)$. We observe that both functors are exact and fully faithful.

Lemma 2.1. Let $\mathcal{A}$ be an abelian category. Then the monomorphism category $\operatorname{Mon}(\mathcal{A})$ is an exact category such that conflations are given by sequences $\alpha^{\prime} \rightarrow \alpha \rightarrow \alpha^{\prime \prime}$ with the induced sequences of sources and targets short exact in $\mathcal{A}$.

Assume further that $\mathcal{A}$ is Frobenius. Then the exact category $\operatorname{Mon}(\mathcal{A})$ is Frobenius such that its projective objects are equal to direct summands of objects of the form $i_{1}(P) \oplus i_{2}(P)$ where $P$ is a projective object in $\mathcal{A}$.

Proof. We observe that $\operatorname{Mon}(\mathcal{A})$ is an additive subcategory of the abelian category $\operatorname{Mor}(\mathcal{A})$ which is closed under extensions by Snake Lemma. Then it is an exact category with conflations induced by short exact sequences in $\operatorname{Mor}(\mathcal{A})$; see Example 4.1 in [23].

Assume now that the abelian category $\mathcal{A}$ is Frobenius. We will show first that objects of the form $i_{1}(P)$ and $i_{2}(P)$ are projective and injective. Recall that for an object $\alpha$ in $\operatorname{Mon}(\mathcal{A})$ we denote by $s(\alpha)$ and $t(\alpha)$ the source and target of $\alpha$, respectively. We have the following natural isomorphisms

$\operatorname{Hom}_{M o n}(\mathcal{A})\left(i_{1}(P), \alpha\right) \simeq \operatorname{Hom}_{\mathcal{A}}(P, t(\alpha))$ and $\operatorname{Hom}_{M o n}(\mathcal{A})\left(i_{2}(P), \alpha\right) \simeq \operatorname{Hom}_{\mathcal{A}}(P, s(\alpha))$. 
These isomorphisms show that the objects $i_{1}(P)$ and $i_{2}(P)$ are projective. Similarly, we have the following natural isomorphisms

$$
\operatorname{Hom}_{\operatorname{Mon}(\mathcal{A})}\left(\alpha, i_{1}(P)\right) \simeq \operatorname{Hom}_{\mathcal{A}}(\operatorname{Cok} \alpha, P)
$$

and

$$
\operatorname{Hom}_{M o n}(\mathcal{A})\left(\alpha, i_{2}(P)\right) \simeq \operatorname{Hom}_{\mathcal{A}}(t(\alpha), P) .
$$

These isomorphisms show that the objects $i_{1}(P)$ and $i_{2}(P)$ are injective; here, we use that the functor Cok of taking the cokernels is exact on $\operatorname{Mon}(\mathcal{A})$ by Snake Lemma.

Let $\alpha$ be an object in $\operatorname{Mon}(\mathcal{A})$. Take epimorphisms $P \rightarrow s(\alpha)$ and $P \rightarrow t(\alpha)$ with $P$ projective in $\mathcal{A}$. Then we have an epimorphism $i_{1}(P) \oplus i_{2}(P) \rightarrow \alpha$ whose kernel lies in $\operatorname{Mon}(\mathcal{A})$. This shows that the exact category $\operatorname{Mon}(\mathcal{A})$ has enough projective objects. On the other hand, for the object $\alpha$, take monomorphisms $a: t(\alpha) \rightarrow P$ and $b^{\prime}:$ Cok $\alpha \rightarrow P$ with $P$ projective in $\mathcal{A}$. Denote by $b$ the composite $t(\alpha) \rightarrow \operatorname{Cok} \alpha \stackrel{b^{\prime}}{\rightarrow} P$ where the first morphism is the natural projection. Consider the following morphism in $\operatorname{Mor}(\mathcal{A})$

$$
\left(\left(\begin{array}{c}
a \circ \alpha \\
0
\end{array}\right),\left(\begin{array}{l}
a \\
b
\end{array}\right)\right): \alpha \longrightarrow i_{2}(P) \oplus i_{1}(P) .
$$

It is a monomorphism and by a diagram-chasing its cokernel lies in $\operatorname{Mon}(\mathcal{A})$. Then it becomes a conflation in $\operatorname{Mon}(\mathcal{A})$. This shows that the exact category $\operatorname{Mon}(\mathcal{A})$ has enough injective objects. From the argument above, it is direct to conclude that in the exact category $\operatorname{Mon}(\mathcal{A})$ the class of projective objects coincides with the class of injective objects, and projective objects are direct summands of objects of the form $i_{1}(P) \oplus i_{2}(P)$ where $P$ is a projective object in $\mathcal{A}$.

Remark 2.2. With a slightly modified proof as above, one can show that a similar result holds if the category $\mathcal{A}$ is an exact category. In this case, one replaces $\operatorname{Mon}(\mathcal{A})$ by the inflation category of $\mathcal{A}$; compare [22, Section 5] and [21].

For a Frobenius abelian category $\mathcal{A}$, we denote by $\underline{\operatorname{Mon}}(\mathcal{A})$ the stable category of $\operatorname{Mon}(\mathcal{A})$ modulo projective objects; it is a triangulated category. We will call it the stable monomorphism category of $\mathcal{A}$.

We observe that both the functors $i_{1}$ and $i_{2}$ are fully faithful and send projective objects to projective objects. Then they induce fully faithful triangle functors $i_{1}: \underline{\mathcal{A}} \rightarrow$ $\underline{\operatorname{Mon}}(\mathcal{A})$ and $i_{2}: \underline{\mathcal{A}} \rightarrow \underline{\operatorname{Mon}}(\mathcal{A})([18$, p.23, Lemma 2.8]).

\section{Tilting Objects in Stable Monomorphism Category}

In this section, we will show that for a Frobenius abelian category $\mathcal{A}$, a tilting object in the stable category $\underline{\mathcal{A}}$ induces naturally a tilting object in the stable monomorphism category $\operatorname{Mon}(\mathcal{A})$. We characterize the stable category of Ringel-Schmidmeier as the bounded derived category of a finite dimensional algebra.

Following Keller, we recall that a triangulated category is algebraical provided that it is triangle equivalent to the stable category of a Frobenius exact category ([24, 
subsection 8.7]). One has a well-behaved notion of tilting object in an algebraical triangulated category.

Let $\mathcal{T}$ be an algebraical triangulated category. Denote by [1] the shift functor and by $[n]$ its $n$-th power for each $n \in \mathbb{Z}$. An object $T$ in $\mathcal{T}$ is a tilting object if the following conditions are satisfied:

(T1) $\operatorname{Hom}_{\mathcal{T}}(T, T[n])=0$ for $n \neq 0$;

(T2) the smallest thick triangulated subcategory of $\mathcal{T}$ containing $T$ is $\mathcal{T}$ itself;

(T3) $\operatorname{End}_{\mathcal{T}}(T)$ is an artin algebra having finite global dimension.

Here, we recall that a triangulated subcategory of $\mathcal{T}$ is called thick if it is closed under taking direct summands. We point out that the notion of tilting object presented here is slightly different from, however closely related to, the ones in [18] and [24].

Recall that an additive category is said to be idempotent-split provided that each idempotent $e: X \rightarrow X$ admits a factorization $X \stackrel{u}{\rightarrow} Y \stackrel{v}{\rightarrow} X$ such that $u \circ v=\operatorname{Id}_{Y}([18$, Chapter I, 3.2]). Recall that for an artin algebra $A$ having finite global dimension, the bounded derived category $\mathbf{D}^{b}(\bmod A)$ is algebraical and idempotent-split (see the proof of [18, Chapter I, Corollary 4.9]), and it has $A_{A}$ as its tilting object.

The following remarkable result due to Keller claims that the converse holds true (compare [9, Theorem 1]).

Lemma 3.1. (Keller) Let $\mathcal{T}$ be an idempotent-split algebraical triangulated category with a tilting object $T$. Then there is a triangle equivalence

$$
\mathcal{T} \simeq \mathbf{D}^{b}\left(\bmod \operatorname{End}_{\mathcal{T}}(T)\right)
$$

Proof. Set $A=\operatorname{End}_{\mathcal{T}}(T)$. By [24, Theorem 8.51 a)] there is a triangle functor $F^{\prime}: \mathcal{T} \rightarrow \mathbf{D}\left(A^{\prime}\right)$ sending $T$ to $A^{\prime}$, where $A^{\prime}$ is a differential graded algebra with the only nonzero cohomology $H^{0}\left(A^{\prime}\right) \simeq A$ and $\mathbf{D}\left(A^{\prime}\right)$ is the (unbounded) derived category of differential graded (right) modules on $A^{\prime}$. By [24, subsection 8.4] there is a triangle equivalence $\mathbf{D}\left(A^{\prime}\right) \simeq \mathbf{D}(\operatorname{Mod} A)$ identifying $A^{\prime}$ with $A_{A}$, where $\operatorname{Mod} A$ is the category of (not necessarily finitely generated) right $A$-modules. Consequently, there is a triangle functor $F: \mathcal{T} \rightarrow \mathbf{D}(\operatorname{Mod} A)$ sending $T$ to $A$. Using (T1) and (T2) and applying Beilinson Lemma ([18, p.72, Lemma 3.4]), the triangle functor $F$ is fully faithful. Then we may view $\mathcal{T}$ as a triangulated subcategory of $\mathbf{D}(\operatorname{Mod} A)$; moreover, since $\mathcal{T}$ is idempotent-split, it is necessarily a thick subcategory of $\mathbf{D}(\operatorname{Mod} A)$. By (T3) the artin algebra $A$ has finite global dimension, and then the smallest thick triangulated subcategory of $\mathbf{D}(\operatorname{Mod} A)$ containing $A_{A}$ is $\mathbf{D}^{b}(\bmod A)$. From this we conclude that the essential image of $F$ is $\mathbf{D}^{b}(\bmod A)$. Therefore $F$ induces the required equivalence.

Our first observation states that a tilting object in the stable category $\underline{\mathcal{A}}$ induces naturally a tilting object in the stable monomorphism category $\operatorname{Mon}(\mathcal{A})$. Recall that for an artin algebra $A, T_{2}(A)=\left(\begin{array}{cc}A & A \\ 0 & A\end{array}\right)$ is the $2 \times 2$ upper triangular matrix algebra ([3, Chapter III, Section 2]). 
Theorem 3.2. Let $\mathcal{A}$ be a Frobenius abelian category such that $T$ is a tilting object in its stable category $\underline{\mathcal{A}}$. Then $T^{\prime}=i_{1}(T) \oplus i_{2}(T)$ is a tilting object in $\underline{\operatorname{Mon}}(\mathcal{A})$; moreover, we have an isomorphism $\operatorname{End}_{\underline{\underline{M n n}}(\mathcal{A})}\left(T^{\prime}\right) \simeq T_{2}\left(\operatorname{End}_{\underline{\mathcal{A}}}(T)\right)$ of algebras.

Proof. Recall that $i_{1}: \underline{\mathcal{A}} \rightarrow \underline{\operatorname{Mon}}(\mathcal{A})$ and $i_{2}: \underline{\mathcal{A}} \rightarrow \underline{\operatorname{Mon}}(\mathcal{A})$ are fully faithful triangle functors. Observe that for objects $A$ and $B$ in $\mathcal{A}, \operatorname{Hom}_{\underline{\operatorname{Mon}}(\mathcal{A})}\left(i_{2}(A), i_{1}(B)\right)=0$. So to check the condition (T1) for $T^{\prime}$, it suffices to show that $\operatorname{Hom}_{\operatorname{Mon}(\mathcal{A})}\left(i_{1}(T), i_{2}(T)[n]\right)=$ 0 for $n \neq 0$. For this end, note that since $i_{2}$ is a triangle functor, we have

$$
i_{2}(T)[n] \simeq i_{2}(T[n])=T[n] \stackrel{\operatorname{Id}_{T[n]}}{\longrightarrow} T[n] .
$$

Thus a morphism in $\operatorname{Hom}_{\operatorname{Mon}(\mathcal{A})}\left(i_{1}(T), i_{2}(T)[n]\right)$ is of the form $(0, f)$, where $f: T \rightarrow$ $T[n]$ is a morphism in $\mathcal{A}$. By the condition (T1) for $T, f$ factors through a projective object $P$ in $\mathcal{A}$. Therefore the morphism $(0, f)$ factors through $i_{1}(P)$, which is projective in $\operatorname{Mon}(\mathcal{A})$; see Lemma 2.1. Hence $(0, f)=0$ in the stable monomorphism category $\underline{\operatorname{Mon}}(\mathcal{A})$.

To check (T2) for $T^{\prime}$, recall that each object $\alpha$ fits into a conflation

$$
i_{2}(s(\alpha)) \longrightarrow \alpha \longrightarrow i_{1}(\operatorname{Cok} \alpha)
$$

and thus into a triangle

$$
i_{2}(s(\alpha)) \longrightarrow \alpha \longrightarrow i_{1}(\operatorname{Cok} \alpha) \longrightarrow i_{2}(s(\alpha))[1] .
$$

Here as in Section 2, $s(\alpha)$ denotes the source of $\alpha$. Hence the smallest triangulated subcategory of $\underline{\operatorname{Mon}}(\mathcal{A})$ containing $i_{1}(\underline{\mathcal{A}})$ and $i_{2}(\underline{\mathcal{A}})$ is $\underline{\operatorname{Mon}}(\mathcal{A})$ itself. Now applying the condition (T2) of $T$, we infer that (T2) holds for $T^{\prime}$.

Finally to see the condition (T3) for $T^{\prime}$, it is direct to check that $\operatorname{End}_{\operatorname{Mon}(\mathcal{A})}\left(T^{\prime}\right) \simeq$ $T_{2}\left(\operatorname{End}_{\mathcal{A}}(T)\right)$. Recall that the algebra $\operatorname{End}_{\mathcal{A}}(T)$ has finite global dimension. Then by $\left[3\right.$, Chapter III, Proposition 2.6] we infer that $\operatorname{End}_{\underline{M o n}(\mathcal{A})}\left(T^{\prime}\right)$ has finite global dimension.

We will give an application of Theorem 3.2. Let $A=\oplus_{n \geq 0} A_{n}$ be a positively graded artin algebra. Denote by $c$ the maximal integer such that $A_{c} \neq 0$. Consider the following upper triangular matrix algebra

$$
\mathrm{b}(A)=\left(\begin{array}{ccccc}
A_{0} & A_{1} & \cdots & A_{c-2} & A_{c-1} \\
0 & A_{0} & \cdots & A_{c-3} & A_{c-2} \\
\vdots & \vdots & \ddots & \vdots & \vdots \\
0 & 0 & \cdots & A_{0} & A_{1} \\
0 & 0 & \cdots & 0 & A_{0}
\end{array}\right) .
$$

Here the multiplication of $\mathrm{b}(A)$ is induced from the one of $A$. This algebra is called the Beilinson algebra of $A$ in [12].

Denote by $\bmod ^{\mathbb{Z}} A$ the category of finitely generated $\mathbb{Z}$-graded $A$-modules with homomorphisms preserving degrees. We say that $A$ is graded self-injective provided that $\bmod ^{\mathbb{Z}} A$ is a Frobenius category. In fact, this is equivalent to that as a ungraded algebra $A$ is self-injective $([15,12])$. In this case, we denote by $\underline{\bmod }^{\mathbb{Z}} A$ the stable category of $\bmod ^{\mathbb{Z}} A$ modulo projective modules; it is a triangulated category. 
We say that a graded algebra $A$ is right well-graded, provided that $A_{c}$, as a right $A_{0}$-module, is sincere in the sense of [3, p.317]. In fact, for a graded self-injective algebra $A$, it is right well-graded if and only if it is left well-graded; see [12, Lemma 2.2 . In this case we will simply say that the graded algebra $A$ is well-graded.

Corollary 3.3. Let $A=\oplus_{n \geq 0} A_{n}$ be a positively graded self-injective artin algebra which is well-graded. Suppose that $A_{0}$ has finite global dimension. Then there is a triangle equivalence

$$
\underline{\operatorname{Mon}}\left(\bmod ^{\mathbb{Z}} A\right) \simeq \mathbf{D}^{b}\left(\bmod T_{2}(\mathrm{~b}(A))\right) .
$$

Proof. By [3, Chapter III, Proposition 2.6], the Beilinson algebra $\mathrm{b}(A)$ and then $T_{2}(\mathrm{~b}(A))$ has finite global dimension. By [12, Corollary 1.2] there is a triangle equivalence $\underline{\bmod }^{\mathbb{Z}} A \simeq \mathbf{D}^{b}(\bmod \mathrm{b}(A))$. In particular, there is a tilting object $T$ in $\bmod ^{\mathbb{Z}} A$ with endomorphism algebra $\mathrm{b}(A)$. We apply Theorem 3.2 to get a tilting object $T^{\prime}$ in $\underline{\operatorname{Mon}}\left(\bmod ^{\mathbb{Z}} A\right)$ whose endomorphism algebra is isomorphic to $T_{2}(\mathrm{~b}(A))$. Note that the stable monomorphism category $\underline{\operatorname{Mon}}\left(\bmod ^{\mathbb{Z}} A\right)$ is idempotent-split; in fact, it is even a Krull-Schmidt category. Then the result follows immediately from Lemma 3.1.

In what follows, we will apply the obtained results to the stable category of RingelSchmidmeier.

Let $k$ be a field and let $p \geq 2$ be an integer. Consider the truncated polynomial algebra $A=k[t] /\left(t^{p}\right)$ with $t$ an indeterminant; it is positively graded such that $\operatorname{deg} t=$ 1. Observe that $A$ is graded self-injective and moreover it is well-graded. In particular, the category $\bmod ^{\mathbb{Z}} A$ of finitely generated graded $A$-modules is Frobenius. Following [36, subsection 0.4], we denote by $\mathcal{S}(\widetilde{p})$ the category of pairs $(V, U)$, where $V$ is a graded module over $A$ and $U \subseteq V$ is a graded submodule, and the morphisms in this category are given by morphisms in the graded module category which respect the inclusion. There is a natural identification $\mathcal{S}(\widetilde{p})=\operatorname{Mon}\left(\bmod ^{\mathbb{Z}} A\right)$ and then by Lemma 2.1 it is a Frobenius exact category. Hence its stable category $\mathcal{S}(\widetilde{p})$ modulo projective objects is triangulated. This triangulated category will be called the stable category of Ringel-Schmidmeier.

We note that the Beilinson algebra $\mathrm{b}(A)$ of the graded algebra $A$ is isomorphic to the path algebra $k \mathbb{A}_{p-1}$ of the linear quiver $\mathbb{A}_{p-1}$ with $p-1$ vertices and linear orientation (compare [33, Example 2.9]). Then the $2 \times 2$ upper triangular matrix algebra $T_{2}(\mathrm{~b}(A))$ is given by the following quiver with $2 p-2$ vertices subject to the commutativity relation

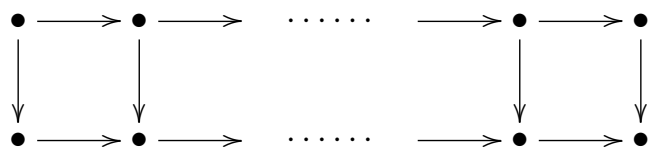

We observe that $T_{2}(\mathrm{~b}(A)) \simeq k \mathbb{A}_{2} \otimes_{k} k \mathbb{A}_{p-1}$. Let us mention that these diagrams and algebras are studied in [28].

Then we have the following immediate consequence of Corollary 3.3. 
Corollary 3.4. Use the notation above. Then there is a triangle equivalence

$$
\underline{\mathcal{S}}(\widetilde{p}) \simeq \mathbf{D}^{b}\left(\bmod k \mathbb{A}_{2} \otimes_{k} k \mathbb{A}_{p-1}\right) .
$$

Remark 3.5. Let us remark that taking into account of the results obtained in [26] and [28, Corollary 1.2], one may find a close relation between Corollary 3.4 and some results in [27].

Recall that $T=\bigoplus_{i=0}^{p-2}\left(A /\left(t^{p-i-1}\right)\right)(i)$ is a tilting object in $\underline{\bmod }^{\mathbb{Z}} A$, where $(i)$ denote the degree-shift functors ([31] and [15]). This assertion can be obtained from the proof of [33, Corollary 2.8] or [12, Corollary 1.2]. We apply Theorem 3.2 to deduce that $T^{\prime}=i_{1}(T) \oplus i_{2}(T)$ is a tilting object in $\underline{\mathcal{S}}(\widetilde{p})$, which yields the triangle equivalence in Corollary 3.4. We point out that this explicit tilting object is also obtained in [27, Lemma 4.7] via a different method.

\section{Stable Monomorphism Category as Singularity Category}

In this section, we will relate the stable monomorphism category of the (graded) module category of a (graded) self-injective algebra to the (graded) singularity category of the associated (graded) $2 \times 2$ upper triangular matrix algebra. We characterize the stable category of Ringel-Schmidmeier as the graded singularity category of a finite dimensional graded algebra.

Let $A$ be an artin algebra. Recall that the bounded homotopy category $\mathbf{K}^{b}(\operatorname{proj} A)$ of projective modules is viewed naturally as a triangulated subcategory of $\mathbf{D}^{b}(\bmod A)$. Following [32, 33], we call the Verdier quotient triangulated category

$$
\mathbf{D}_{\mathrm{sg}}(A)=\mathbf{D}^{b}(\bmod A) / \mathbf{K}^{b}(\operatorname{proj} A)
$$

the singularity category of $A$; compare [10] and [19].

Recall that for an artin algebra $A, T_{2}(A)$ is the $2 \times 2$ upper triangular matrix algebra of $A$. We consider the following composite functor

$$
G_{A}: \operatorname{Mon}(\bmod A) \hookrightarrow \bmod T_{2}(A) \longrightarrow \mathbf{D}^{b}\left(\bmod T_{2}(A)\right) \longrightarrow \mathbf{D}_{\mathrm{sg}}\left(T_{2}(A)\right) .
$$

Here, the first inclusion is obtained by regarding morphisms in mod $A$ as (right) $T_{2}(A)$ modules ([3, Chapter III, Proposition 2.2]), the middle functor identifies modules with stalk complexes concentrated at degree zero ([20, p.40, Proposition 4.3]), and the last functor is the quotient functor.

Our second observation is as follows.

Theorem 4.1. Let $A$ be a self-injective algebra. Then the functor $G_{A}$ induces a triangle equivalence

$$
\underline{\operatorname{Mon}}(\bmod A) \simeq \mathbf{D}_{\mathrm{sg}}\left(T_{2}(A)\right) .
$$

Before giving the proof, we recall several notions. Let $A$ be an artin algebra. Following [5, p.400], an acyclic complex $P^{\bullet}$ of projective $A$-modules is called totally acyclic if the Hom complex $\operatorname{Hom}_{A}\left(P^{\bullet}, A\right)$ is still acyclic (also see [25, Section 7]). An $A$-module $M$ is said to be Gorenstein-projective if there is a totally acyclic complex $P^{\bullet}$ such that its zeroth cocycle $Z^{0}\left(P^{\bullet}\right)$ is isomorphic to $M([16$, Chapter 10]). Recall that a module 
$M$ is Gorenstein-projective if and only if $\operatorname{Ext}_{A}^{i}(M, A)=0, \operatorname{Ext}_{A \text { op }}^{i}\left(\operatorname{Hom}_{A}(M, A), A\right)=$ 0 for $i \geq 1$ and the natural map $M \rightarrow \operatorname{Hom}_{A^{\text {op }}}\left(\operatorname{Hom}_{A}(M, A), A\right)$ is an isomorphism (compare [14, Definition (1.1.2)]).

We denote by Gproj $A$ the full subcategory of $\bmod A$ consisting of Gorensteinprojective modules. Observe that projective modules are Gorenstein-projective and thus proj $A \subseteq$ Gproj $A$. Moreover, by [2, Proposition 5.1] the subcategory Gproj $A$ is closed under extensions and taking direct summands (also see [16]), and then it is direct to see that Gproj $A$ is a Frobenius exact category such that its projective objects are equal to projective $A$-modules ([6, Proposition 3.8(i)] and [13, Proposition $3.1(1)])$. Denote by Gproj $A$ its stable category modulo projective $A$-modules; it is a triangulated category.

Recall that an artin algebra $A$ is said to be Gorenstein if the regular modules ${ }_{A} A$ and $A_{A}$ have finite injective dimensions ([19]). In this case the two dimensions are equal and the common value is denoted by G.dim $A$. We say that the Gorenstein algebra $A$ is 1 -Gorenstein provided that G.dim $A \leq 1$.

For an artin algebra $A$, denote by sub $A$ the full subcategory of mod $A$ consisting of submodules of projective modules; these modules are called torsionless modules. We remark that homological properties of torsionless modules are studied in [4].

The following result is well known.

Lemma 4.2. Let $A$ be a 1-Gorenstein algebra. Then we have Gproj $A=\operatorname{sub} A$.

Proof. The inclusion Gproj $A \subseteq \operatorname{sub} A$ is easy. On the other hand, assume that $M$ is a torsionless module. Consider a short exact sequence $0 \rightarrow M \rightarrow P \rightarrow M^{\prime} \rightarrow 0$ with $P$ projective. Since the regular module $A_{A}$ has injective dimension at most one, using dimension shift, we infer that $\operatorname{Ext}^{i}(M, A)=0$ for $i \geq 1$. Then by [16, Corollary 11.5.3] (see also [13, Lemma 3.7] and [25, Proposition 7.13]), $M$ is Gorenstein-projective.

The next observation is essentially due to Li and Zhang ([30, Theorem 1.1]; also see [7, Example 4.17] and [21, Proposition 3.6]). Recall that for an artin algebra $A$, a morphism of (right) $A$-modules is identified with a (right) module over $T_{2}(A)$; in fact, this yields an equivalence $\operatorname{Mor}(\bmod A) \simeq \bmod T_{2}(A)$ of categories; see [3, Chapter III, Proposition 2.2].

Lemma 4.3. Let $A$ be a self-injective algebra. Then we have an equivalence of categories

$$
\operatorname{Mon}(\bmod A) \simeq \operatorname{sub} T_{2}(A) .
$$

Proof. Recall the equivalence $\operatorname{Mor}(\bmod A) \simeq \bmod T_{2}(A)$. Observe that the regular module $T_{2}(A)_{T_{2}(A)}$ corresponds to the monomorphism $\left(\begin{array}{c}0 \\ \operatorname{Id}_{A}\end{array}\right): A \rightarrow A \oplus A$. From this one infers that torsionless $T_{2}(A)$-modules correspond to monomorphisms in mod $A$. On the other hand, the third paragraph of the proof of Lemma 2.1 already shows that for a monomorphism $\alpha$, there is a short exact sequence $0 \rightarrow \alpha \rightarrow\left(\begin{array}{c}0 \\ \operatorname{Id}_{P}\end{array}\right) \rightarrow \alpha^{\prime} \rightarrow 0$ in $\operatorname{Mor}(\bmod A)$ such that $P$ is a projective $A$-module. Observe that the monomorphism $\left(\begin{array}{c}0 \\ \operatorname{Id}_{P}\end{array}\right)$ corresponds to a projective $T_{2}(A)$-module. Therefore the monomorphism $\alpha$ corresponds to a torsionless $T_{2}(A)$-module. This completes the proof. 
We will recall the last ingredient in our proof. Let $A$ be an artin algebra. Consider the following composite of functors

$$
F_{A}: \operatorname{Gproj} A \hookrightarrow \bmod A \longrightarrow \mathbf{D}^{b}(\bmod A) \longrightarrow \mathbf{D}_{\mathrm{sg}}(A)
$$

where from the left side, the first functor is the inclusion, the second identifies modules with stalk complexes concentrated in degree zero ([20, p.40, Proposition 4.3]) and the last is the quotient functor. Observe that the additive functor $F_{A}$ vanishes on projective modules and then induces uniquely an additive functor Gproj $A \rightarrow \mathbf{D}_{\mathrm{sg}}(A)$, which is still denoted by $F_{A}$.

The following important result is due to Buchweitz ([10, Theorem 4.4.1]) and independently due to Happel ([19, Theorem 4.6]); also see [13, Proposition 3.5 and Theorem 3.8].

Lemma 4.4. (Buchweitz-Happel) Let $A$ be an artin algebra. Then the functor $F_{A}:$ Gproj $A \rightarrow \mathbf{D}_{\mathrm{sg}}(A)$ is a fully faithful triangle functor. Moreover, if $A$ is Gorenstein, then the functor $F_{A}$ is dense and thus a triangle equivalence.

Proof of Theorem 4.1. We observe that by [11, Remark 3.5] (also see [17, 19]) the algebra $T_{2}(A)$ is 1-Gorenstein and then we can apply Lemma 4.2. Then Lemma 4.3 yields an equivalence of categories $\operatorname{Mon}(\bmod A) \simeq \operatorname{Gproj} T_{2}(A)$. We observe that this equivalence preserves the exact structures, that is, the equivalence and its quasiinverse preserve short exact sequences in $\operatorname{Mon}(\bmod A)$ and $\operatorname{Gproj} T_{2}(A)$. Therefore, this equivalence is an equivalence of Frobenius exact categories. Consequently, we have an induced equivalence of triangulated categories

$$
\underline{\operatorname{Mon}}(\bmod A) \simeq \underline{\operatorname{Gproj}} T_{2}(A) .
$$

Then the result follows directly from Lemma 4.4 .

We will need a graded version of Theorem 4.1. Let $A=\oplus_{n \geq 0} A_{n}$ be a positively graded artin algebra. Denote by $\operatorname{proj}^{\mathbb{Z}} A$ the full subcategory of $\bmod ^{\mathbb{Z}} A$ consisting of projective objects. Following [33], one has the graded singularity category of $A$ defined by

$$
\mathbf{D}_{\mathrm{sg}}^{\mathbb{Z}}(A)=\mathbf{D}^{b}\left(\bmod ^{\mathbb{Z}} A\right) / \mathbf{K}^{b}\left(\operatorname{proj}^{\mathbb{Z}} A\right)
$$

For a graded module $M=\oplus_{i \in \mathbb{Z}} M_{i}$ and an integer $d \in \mathbb{Z}$, its shifted module $M(d)$ has the same module structure as $M$ while it is graded such that $M(d)_{i}=M_{d+i}$ for all $i \in \mathbb{Z}$. This defines automorphisms $(d): \bmod ^{\mathbb{Z}} A \rightarrow \bmod ^{\mathbb{Z}} A$, which are called degree-shift functors. For graded modules $M, N$, we write $\operatorname{HOM}_{A}(M, N)=$ $\oplus_{i \in \mathbb{Z}} \operatorname{Hom}_{\bmod ^{\mathbb{Z}}{ }_{A}}(M, N(i))$ and set $\operatorname{EXT}_{A}^{n}(-,-)$ to be the $n$-th right derived functors ([31] and [15]).

An acyclic complex $P^{\bullet}$ in proj ${ }^{\mathbb{Z}} A$ is totally acyclic if the complex $\operatorname{HOM}_{A}\left(P^{\bullet}, A\right)$ in $\operatorname{proj}^{\mathbb{Z}} A^{\text {op }}$ is acyclic. A graded $A$-module is called graded Gorenstein-projective provided that it is the zeroth cocycle of a totally acyclic complex. Thus we have a full subcategory Gproj ${ }^{\mathbb{Z}} A$ of $\bmod ^{\mathbb{Z}} A$ consisting of graded Gorenstein-projective modules and evidently proj ${ }^{\mathbb{Z}} A \subseteq$ Gproj $^{\mathbb{Z}} A$. As in the ungraded case, the category $\operatorname{Gproj}^{\mathbb{Z}} A$ is a Frobenius exact category with its projective objects equal to graded projective $A$-modules. 
Recall that a graded artin algebra $A$ is said to be graded Gorenstein if the graded regular modules ${ }_{A} A$ and $A_{A}$ have finite injective dimensions in $\bmod ^{\mathbb{Z}} A$ and $\bmod ^{\mathbb{Z}} A^{\mathrm{op}}$, respectively. In this case the two dimensions are the same, which will be denoted by G.dim $\mathbb{Z}^{\mathbb{Z}} A$.

We observe the following fact, which guarantees in principle that most results in Gorenstein homological algebra hold true in the graded situation.

Lemma 4.5. Let $A$ be a positively graded artin algebra, and let $M$ be a graded $A$ module. Then we have

(1) the module $M$ is graded Gorenstein-projective if and only if it is Gorensteinprojective as a ungraded module;

(2) the algebra $A$ is graded Gorenstein if and only if it is Gorenstein as a ungraded algebra; in this case, we have G.dim ${ }^{\mathbb{Z}} A=\mathrm{G} \cdot \operatorname{dim} A$.

Proof. For (1), it suffices to recall that a graded module $M$ is graded Gorensteinprojective if and only if $\operatorname{EXT}_{A}^{i}(M, A)=0, \operatorname{EXT}_{A^{\text {op }}}^{i}\left(\operatorname{HOM}_{A}(M, A), A\right)=0$ for $i \geq 1$ and the natural map $M \rightarrow \mathrm{HOM}_{A^{\text {op }}}\left(\operatorname{HOM}_{A}(M, A), A\right)$ is an isomorphism of graded modules; moreover, for graded modules $M$ and $N$ we have for each $i$ a natural identification $\operatorname{EXT}_{A}^{i}(M, N)=\operatorname{Ext}_{A}^{i}(M, N)$ ([31, Corollary 2.4.7]). For (2), we observe that a graded module $M$ has finite injective dimension in $\bmod ^{\mathbb{Z}} A$ if and only if it has finite injective dimension as a ungraded module; moreover, the two dimensions are the same ([31, Theorem 2.8.7]).

One can show the graded analogues of Lemmas 4.2, 4.3 and 4.4. Using these, we have the following graded analogue of Theorem 4.1.

Proposition 4.6. Let $A=\oplus_{n \geq 0} A_{n}$ be a positively graded self-injective artin algebra. Denote by $T_{2}(A)$ the $2 \times 2$ upper triangular matrix algebra of $A$ which is graded such that $T_{2}(A)_{n}=T_{2}\left(A_{n}\right)$ for $n \geq 0$. Then we have a triangle equivalence

$$
\underline{\operatorname{Mon}}\left(\bmod ^{\mathbb{Z}} A\right) \simeq \mathbf{D}_{\mathrm{sg}}^{\mathbb{Z}}\left(T_{2}(A)\right)
$$

We apply Proposition 4.6 to the stable category of Ringel-Schmidmeier.

Let $k$ be a field and $p \geq 2$ be an integer. Recall from Section 3 that $A=k[t] /\left(t^{p}\right)$ with $\operatorname{deg} t=1$, which is graded self-injective. We observe that $T_{2}(A)$ is isomorphic, as a graded algebra, to $T_{2}(k)[t] /\left(t^{p}\right)$, while the latter is graded such that $\operatorname{deg} T_{2}(k)=0$ and $\operatorname{deg} t=1$.

Recall that the category $\mathcal{S}(\widetilde{p})$ is identified with $\operatorname{Mon}\left(\bmod ^{\mathbb{Z}} A\right)$, and then the stable category $\underline{\mathcal{S}}(\widetilde{p})$ of Ringel-Schmidmeier is identified with $\underline{\operatorname{Mon}}\left(\bmod ^{\mathbb{Z}} A\right)$. Then the following is an immediate consequence of Proposition 4.6.

Corollary 4.7. Use the notation above. Then there is a triangle equivalence

$$
\underline{\mathcal{S}}(\widetilde{p}) \simeq \mathbf{D}_{\mathrm{sg}}^{\mathbb{Z}}\left(T_{2}(k)[t] /\left(t^{p}\right)\right) .
$$


Acknowledgements This research was carried out during the author's visit at the University of Paderborn with a support by Alexander von Humboldt Stiftung. He would like to thank Henning Krause and the faculty of Institut für Mathematik for their hospitality.

The author is indebted to the referee for many helpful suggestions, to Henning Krause for illuminating discussions and to Bernhard Keller and Zhi-Wei Li for helpful comments. The author also would like to thank Dirk Kussin for a private communication. In fact, this work is inspired by a talk given by Dirk Kussin at the University of Paderborn.

\section{References}

[1] D.M. Arnold, Abelian Groups and Representations of Finite Partially Ordered Sets, Springer GMS Books in Mathematics, 2000.

[2] M. Auslander and I. Reiten, Applications of contravariantly finite subcategories, Adv. Math. 86 (1)(1991), 111-152.

[3] M. Auslander, I. Reiten and S.O. Smal $\varnothing$, Representation Theory of Artin Algebras, Cambridge Univ. Press, 1995.

[4] M. Auslander and S.O. Smal $\varnothing$, Almost split sequences in subcategories, J. Algebra 69 (1981), 426-454.

[5] L.L. Avramov and A. Martsinkovsky, Absolute, relative, and Tate cohomology of modules of finite Gorenstein dimension, Proc. London Math. Soc. (3) 85 (2002), no.2, 393-440

[6] A. Beligiannis, Cohen-Macaulay modules, (co)torsion pairs and virtually Gorenstein algebras, J. Algebra 288 (2005), 137-211.

[7] A. Beligiannis, On algebras of finite Cohen-Macaulay type, Adv. Math. (2010), doi:10.1016/j.aim.2010.09.006.

[8] G. Birkhoff, Subgroups of abelian groups, Proc. London Math. Soc. II, Ser. 38 (1934), 385401.

[9] A.I. Bondal And M.M. Kapranov, Enhanced triangulated categories, Math. USSR Sbornik 70 (1990), no.1, 93-107.

[10] R.O. Buchweitz, Maximal Cohen-Macaulay Modules and Tate Cohomology over Gorenstein Rings, Unpublished manuscript, 155pp (1987). Available at https://tspace.library.utoronto.ca/handle/1807/16682.

[11] X.W. Chen, Singularity categories, Schur functors and triangular matrix rings, Algebra and Representation Theory 12 (2009), 181-191.

[12] X.W. Chen, Graded self-injective algebras "are" trivial extensions, J. Algebra 322 (2009), 2601-2606.

[13] X.W. Chen, Relative singularity categories and Gorenstein-projective modules, Math. Nachr., to appear, arXiv: 0709.1762v1.

[14] L.W. Christensen, Gorenstein Dimension, Lecture Notes in Math. 1747, Springer-Verlag, Berlin Heidelberg, 2000.

[15] R. Gordon and E.L. Green, Graded Artin algebras, J. Algebra 76(1) (1982), 111-137.

[16] E.E. Enochs And O.M.G. Jenda, Relative Homological Algebra, de Gruyter Expositions in Math. 30, Walter de Gruyter, Berlin New York, 2000.

[17] R.M. Fossum, P. Griffith and I. Reiten, Trivial Extensions of Abelian Categories, Lecture Notes in Math. 456, Springer-Verlag, Berlin Heidelberg New York, 1975.

[18] D. HAppel, Triangulated Categories in the Representation Theory of Finite Dimensional Algebras, London Mathematical Society Lecture Note Ser. 119, Cambridge Univ. Press, 1988

[19] D. Happel, On Gorenstein algebras, Progress in Math., vol. 95, 389-404, Birkhäuser Verlag, Basel, 1991.

[20] R. Hartshorne, Residue and Duality, Lecture Notes in Math. 20, Springer-Verlag, 1966.

[21] O. Iyama, K. Kato And J.I. Miyachi, Recollement of homotopy categories and CohenMacaulay modules, J. K-Theory, to appear; arXiv:0911.0172v1.

[22] B. Keller, Chain complexes and stable categories, Manuscripta Math. 67 (1990), 379-417. 
[23] B. Keller, Derived categories and their uses, in: Handbook of Algebra 1, 671-701, NorthHolland, Amsterdam, 1996.

[24] B. Keller, Derived categories and tilting, in: Handbook of Tilting Theory, 49-104, London Math. Soc. Lecture Note Ser. 332, Cambridge Univ. Press, Cambridge, 2007.

[25] H. Krause, The stable derived category of a noetherian scheme, Compositio Math. 141 (2005), $1128-1162$.

[26] D. Kussin, H. Lenzing and H. Meltzer, Triangle singularities, ADE-chains and weighted projective lines, preprint, 2009.

[27] D. Kussin, H. Lenzing And H. Meltzer, Categories of vector bundles and invariant subspaces of nilpotent operators, arXiv:1002.3797v1.

[28] S. LADKANI, On derived equivalences of lines, rectangles and triangles, arXiv:0911.5137v1.

[29] H. Lenzing, Hereditary categories, in: Handbook of Tilting Theory, 105-146, London Math. Soc. Lecture Note Ser. 332, Cambridge Univ. Press, Cambridge, 2007.

[30] Z.W. Li And P. Zhang, A construction of Gorenstein-projective modules, J. Algebra 323 (6) (2010), 1802-1812.

[31] C. Nastasescu and F. Van Oystaeyen, Methods of Graded Rings, Lecture Note in Math. 1836, Springer, 2004.

[32] D. ORLOv, Triangulated categories of singularities and D-branes in Landau-Ginzburg models, Proc. Steklov Inst. Math. 246 (3) (2004), 227-248.

[33] D. OrLov, Derived categories of coherent sheaves and triangulated categories of singularities, in: Algebra, arithmetic, and geometry: in honor of Yu. I. Manin. Vol. II, 503-531, Progr. Math. 270, Birkhäuser Boston, Inc., Boston, MA, 2009.

[34] C.M. Ringel And M. Schmidmeier, Submodule categories of wild representation type, Jour. Pure and Applied Algebra 205 (2) (2006), 412-422.

[35] C.M. Ringel And M. Schmidmeier, The Auslander-Reiten translation in submodule categories, Trans. Amer. Math. Soc. 360 (2008), 691-716.

[36] C.M. Ringel And M. Schmidmeier, Invariant subspaces of nilpotent operators I, Journal Reine Angew. Math. Band 614 (2008), 1-52.

[37] D. Simson, Chain categories of modules and subprojective representations of posets over uniserial algebras, Rocky Mountain J. Math. 32 (2002), 1627-1650.

Department of Mathematics, University of Science and Technology of China, Hefei 230026, P. R. CHINA

HomePaGe: HTtP://MAIL.USTC.EDU.CN $/ \sim$ XWCHEN

E-mail address: xwchen@mail.ustc.edu.cn 